\title{
Doença de Alzheimer: impacto na qualidade de vida do idoso e cuidador
}

\author{
Alzheimer's disease: impact on elderlys's and caregiver's quality of life \\ Enfermedad de Alzheimer: impacto en la calidad de la deuda
}

Recebido: 16/02/2021 | Revisado: 21/02/2021 | Aceito: 26/02/2021 | Publicado: 04/03/2021

\author{
Ingrid de Sousa Matias \\ ORCID: https://orcid.org/0000-0003-4857-3206 \\ Faculdade Santa Maria, Brasil \\ E-mail: ingridmatias321@gmail.com \\ Mariana Gomes Pinto \\ ORCID: https://orcid.org/0000-0002-6923-7757 \\ Faculdade Santa Maria, Brasil \\ E-mail: marianagomespintto@gmail.com \\ Ingryd Ludmyla Costa dos Santos Sarmento \\ ORCID: https://orcid.org/0000-0002-2012-6140 \\ Faculdade Santa Maria, Brasil \\ E-mail: ingrydcostasarmento@gmail.com \\ Jéssica Alves Moreira \\ ORCID: https://orcid.org/0000-0001-5088-7066 \\ Faculdade Santa Maria, Brasil \\ E-mail: jessica.alvesmoreira@hotmail.com \\ Rafael Barbosa de Moura \\ ORCID: https://orcid.org/0000-0001-8874-5995 \\ Universidade Federal do Cariri, Brasil \\ E-mail: rafael.b.moura@hotmail.com \\ Vanessa Erika Abrantes Coutinho \\ ORCID: https://orcid.org/0000-0002-5473-972X \\ Faculdade Santa Maria, Brasil \\ E-mail: vanessaerika.bio@gmail.com
}

\begin{abstract}
Resumo
Objetivo: Analisar a repercussão do diagnóstico da Doença de Alzheimer (DA) na qualidade de vida do idoso e de seus cuidadores. Métodos: Trata-se de uma revisão integrativa de literatura baseada na pergunta norteadora: Quais os desafios encontrados por pacientes com Alzheimer e seus cuidadores? Foram selecionados artigos científicos, publicados entre 2012 e 2018, nas bases de dados Biblioteca Virtual em Saúde (BVS) e Scientific Electronic Library Online (SciELO). Os descritores "Doença de Alzheimer", "Cuidadores", "Idoso" e o operador booleano AND foram utilizados para a busca. Foram selecionados dezoito artigos científicos disponíveis na íntegra nas bases de dados mencionadas, com idioma em português, inglês ou espanhol e publicação durante o período proposto. Foram excluídos resumos, teses e monografias. Resultados: Os estudos analisados demonstram que o diagnóstico de Doença de Alzheimer (DA) envolve situações desgastantes, já que o declínio cognitivo dos idosos altera o cotidiano familiar e requer assistência integral dos cuidadores, os quais, frequentemente, sentem-se sobrecarregados e têm maior risco de apresentar problemas de saúde. Conclusões: O processo de cuidar na Doença de Alzheimer (DA) tem impacto na qualidade de vida não só dos pacientes com DA, mas também de seus cuidadores e familiares. Considerando a tendência de envelhecimento das populações, trata-se de um cenário desafiador que requer visão holística sobre a condição dos idosos e de seus acompanhantes.
\end{abstract}

Palavras-chave: Doença de Alzheimer; Cuidadores; Idoso.

\begin{abstract}
Objective: Analyze the repercussion of the diagnosis of Alzheimer's Disease on quality of life of the elderly and their caregivers. Methods: This is an integrative review of the literature based on the guiding question: What are the challenges faced by patients with Alzheimer's Disease and their caregivers? Scientific articles were selected, published between 2012 and 2018, on the Virtual Health Library (VHL) and Scientific Eletronic Library Online (SCIELO). The descriptors "Alzheimer's Disease", "Caregivers"," Aged" and boolean operator AND were used for the search. Were selected eighteen scientific articles available in full in the mentioned databases, with language in portuguese, english or spanish and publication during the proposed period. Were excluded abstracts, theses and monographs. Results: The analyzed studies demonstrated that the diagnosis of Alzheimer's Disease (AD) involves stressful situations, as cognitive decline of the elderly changes daily life of the family and requires complete assistance of caregivers, that, frequently, feel overloaded and have higher risk of health problems. Conclusions: The care process in Alzheimer's Disease (AD) has an impact on the quality of life of Alzheimer's patients, their caregivers
\end{abstract}


and relatives. Seeing the trend of population ageing, this is a challenging setting, that requires holistic view of the condition of the elderly and their caregivers.

Keywords: Alzheimer's disease; Caregivers; Aged.

\section{Resumen}

Objetivo: Analizar el impacto del diagnóstico de la Enfermedad de Alzheimer (EA) en la calidad de vida de las personas mayores y sus cuidadores. Métodos: Se trata de una revisión integradora de la literatura basada en la pregunta orientadora: ¿Cuáles son los desafíos que enfrentan los pacientes con Alzheimer y sus cuidadores? Los artículos científicos, publicados entre 2012 y 2018, fueron seleccionados en las bases de datos de la Biblioteca Virtual en Salud (BVS) y de la Biblioteca Electrónica Científica en Línea (SciELO). Los descriptores "Enfermedad de Alzheimer", "Cuidadores", “Anciano" y el operador booleano Y se utilizaron para la búsqueda. Se seleccionaron dieciocho artículos científicos disponible em su totalidade en las bases de datos mencionadas, con idioma en português, inglés o español y publicado durante el período propuesto. Se excluyeron los resúmenes, tesis y monografías. Resultados: Los estudios analizados demuestran que el diagnóstico de la Enfermedad de Alzheimer (EA) involucra situaciones estresantes, ya que el deterioro cognitivo del anciano altera la vida cotidiana de la familia y requiere la asistencia total de los cuidadores, que a menudo se sienten abrumados y corren mayor riesgo de tener problemas de salud. Conclusiones: El proceso de atención de la enfermedad de Alzheimer (EA) impacta en la calidad de vida no solo de los pacientes con EA, sino también de sus cuidadores y parientes. Considerando la tendencia del envejecimiento de la población, se trata de un escenario desafiante, que requiere una visión holística de la condición de los ancianos y sus acompañantes.

Palabras clave: Enfermedad de Alzheimer; Cuidadores; Anciano.

\section{Introdução}

A cada ano cresce o número de idosos nos países desenvolvidos. Segundo o IBGE estima-se que a população com idade superior a 65 anos aumente consideravelmente (cerca de 25,49\%) até 2060.Aliado ao prolongamento da expectativa de vida, observa-se também o aumento da prevalência de doenças neurológicas, dentre as quais, destaca-se a Doença do Alzheimer (DA).

A Doença de Alzheimer (DA) é caracterizada pela perda progressiva de funções cognitivas, como aprendizagem, memória e atenção; que impacta de forma negativa na qualidade das atividades de vida diária, ocupacional e sociais da pessoa (Cardoso et al., 2015). Ela é a principal causa de dependência funcional, institucionalização e morte na terceira idade (Qiu, 2012).

O diagnóstico clínico da doença é realizado através de exclusão (Nitzsche, Moraes \& Júnior, 2015). A avaliação do estado mental busca identificar o comprometimento de aspectos do sistema cognitivo como memória, linguagem, atenção seletiva e dividida, além das funções executivas. Essas informações podem ser obtidas através de um exame direto com o indivíduo acometido ou por meio de entrevista feita com o cuidador. Além disso, o diagnóstico diferencial pode ser feito por meio de exames de imagem cerebral como tomografia computadorizada ou ressonância magnética, para excluir lesões estruturais que possam contribuir para demência (Brasil, 2013).

Considerando o processo de senescência e o caráter progressivo e incapacitante da DA, nota-se que há necessidade de um provedor de cuidado, que deve auxiliar o idoso na realização de suas atividades diárias. A assistência prestada envolve o auxílio na higiene pessoal, alimentação, deambulamento e no acompanhamento aos serviços de saúde (Menezes et al., 2013).

À medida que aumenta a dependência funcional da pessoa acometida por DA, a responsabilidade de cuidar pode sofrer mudanças que, muitas vezes, exigem um maior esforço para suprir as necessidades geradas pela diminuição da função, causando ao cuidador maior estado de vulnerabilidade, desgaste físico, psicológico e/ou social. As queixas mais frequentes desse grupo são sobrecarga, estresse, ansiedade e depressão. Ao prescindir de lazer, vida profissional e até mesmo autocuidado, em função do processo de cuidado, as pessoas que auxiliam os idosos com DA apresentam má qualidade de vida, o que impacta no serviço prestado (Cesário et al., 2017). 


\section{Metodologia}

Trata-se de uma revisão integrativa de literatura de caráter quantitativo. Nesse método de pesquisa, faz-se uma coleta de dados quantitativos de modo que o conjunto gerado pode ser analisado através de critérios numéricos e analíticos aplicáveis ao fenômeno em estudo (Pereira et al., 2018).

A construção da revisão integrativa segue seis diferentes etapas, as quais foram utilizadas nesse estudo: 1. Identificação do tema ou questão de estudo; 2. Estabelecimento de critérios para inclusão e exclusão de estudos; 3 . Definição das informações a serem extraídas dos estudos selecionados; 4. Análise crítica dos estudos; 5. Interpretação dos resultados obtidos com a revisão; 6. Elaboração da síntese do conhecimento.

O estudo foi guiado por uma pergunta norteadora: quais os desafios encontrados por pacientes com Alzheimer e seus cuidadores?

A pesquisa bibliográfica foi realizada por meio da identificação e escolha dos estudos nas seguintes bases de dados: Biblioteca Virtual em Saúde (BVS) e Scientific Electronic Library Online (SCIELO). Os descritores utilizados foram: Doença de Alzheimer, Cuidador, Idoso. Para a busca, também foi utilizado o operador booleano AND. O resultado referente à quantidade de artigos encontrados em cada base de dados, de acordo com os descritores, encontra-se exposto, detalhadamente, na Tabela 1.

Tabela 1 - Número de artigos encontrados nas bases de dados com os descritores da pesquisa.

\begin{tabular}{|l|c|c|}
\hline \multicolumn{1}{|c|}{ Descritores } & Bases de Dados & $\mathbf{N}^{\mathbf{0}}$ de artigos \\
\hline $\begin{array}{l}\text { Alzheimer AND Idosos AND } \\
\text { Cuidadores }\end{array}$ & BVS & \\
\hline $\begin{array}{l}\text { Alzheimer AND Idosos AND } \\
\text { Cuidadores }\end{array}$ & SCIELO & 68 \\
\hline TOTAL & & 1.147 \\
\hline
\end{tabular}

Fonte: Dados da pesquisa (2019).

Observa-se que, através dos descritores Alzheimer AND Idosos AND Cuidadores, foram encontrados 1.147 artigos na base de dados BVS e 68 artigos na base de dados SCIELO, totalizando 1.215 artigos. Para seleção dos artigos científicos condizentes com o propósito do estudo, dentre os encontrados, foram utilizados os seguintes critérios de inclusão: publicação entre 2012 a 2018, trabalhos disponíveis na íntegra, gratuitos, em português, inglês e espanhol, artigos de intervenção, quase experimental, análises, estudo de caso e estudos transversais. Foram excluídos do estudo resumos, teses, dissertações e monografias.

\section{Resultados}

Realizada a busca, conforme os critérios estabelecidos, a amostra foi representada por um total de 18 artigos, cujos dados foram tabulados conforme a tabela 2, onde mostra de forma sequencial a descrição dos artigos de acordo com o(s) autor(es), ano de publicação, título e objetivo(s). Todos os artigos que compõe a amostra foram publicados entre 2012 e 2018. 
Tabela 2 - Informações à cerca dos artigos selecionados para a pesquisa com relação as suas respectivas autorias, ano de publicação e títulos.

\begin{tabular}{|c|c|c|}
\hline $\begin{array}{c}\text { Autor (es) do } \\
\text { Artigo e ano de } \\
\text { publicação }\end{array}$ & Título do Artigo & $\overline{\text { Objetivo(s) }}$ \\
\hline $\begin{array}{l}\text { Pereira, } \\
\text { Teixeira \& } \\
\text { Santos (2012) }\end{array}$ & $\begin{array}{l}\text { Qualidade de vida: abordagens, } \\
\text { conceitos e avaliação }\end{array}$ & $\begin{array}{l}\text { A partir da leitura, discussão e análise da literatura especializada, } \\
\text { apresentar as principais abordagens, conceitos e propostas de classificação } \\
\text { e avaliação da qualidade de vida. }\end{array}$ \\
\hline
\end{tabular}

Cavalcanti \& Aspectos da fisiopatologia da Mostrar a associação das várias causas subjacentes ao processo

Engelhardt Doença de Alzheimer esporádica fisiopatológico da DA, com vistas ao desenvolvimento de marcadores biológicos e estratégias terapêuticas.

\section{Cardoso et al. A Doença de Alzheimer em idosos Identificar, nas produções científicas nacionais, as consequências do e as consequências para cuidadores domiciliares cuidado ao idoso portador de Doença de Alzheimer para os cuidadores domiciliares.}

\section{Carretta \& Perspectivas atuais na prevenção da}

Scherer (2012) Doença de Alzheimer

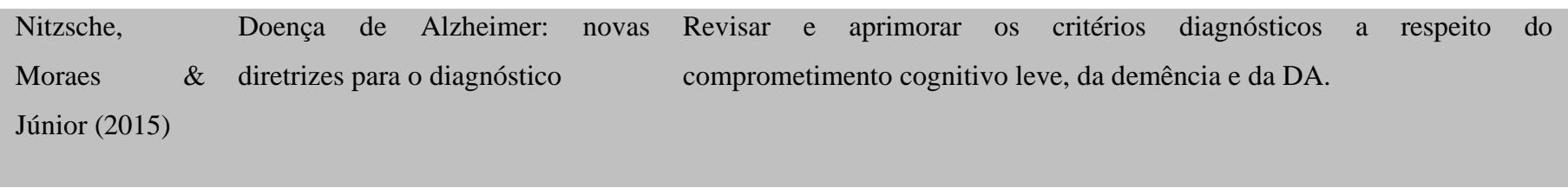

Coelho et al. Os impactos na saúde do cuidador familiar de pessoas com Doença de Alzheimer: uma revisão de Identificar os impactos na saúde do cuidador familiar de pessoas com danos decorrentes do processo de cuidar.

literatura.

$\begin{array}{ll}\text { Santos \& } & \text { Percepção da funcionalidade nas } \\ \text { Borges (2015) } & \text { fases leve e moderada da Doença } \\ & \text { de Alzheimer: visão do paciente e } \\ & \text { seu cuidador }\end{array}$

Neumann \& Doença de Alzheimer: o que muda

Dias (2013) na vida do familiar cuidador?

$\begin{array}{llc}\text { Folle, Shimizy } & \text { Representação social da Doença de } \\ \text { \& Naves (2016) } & \text { Alzheimer para familiares } \\ & \text { cuidadores: desgastante e } \\ & \text { gratificante } & \end{array}$

Cesário et al. Estresse e qualidade de vida do (2017)

\begin{abstract}
Avaliar e comparar a percepção da funcionalidade de idosos com doença de Alzheimer (DA) em relação à percepção de seus cuidadores, bem como avaliar e comparar essa relação de acordo com o grau de comprometimento cognitivo desses idosos.
\end{abstract}

Investigar as mudanças psicossociais que ocorreram na vida de familiares cuidadores de idosos portadores da doença de Alzheimer.

Compreender o conteúdo da Representação Social (SR) de cuidadores familiares de pacientes com Doença de Alzheimer. Analisar a relação entre o estresse e a qualidade de vida do cuidador familiar de idosos portadores da doença de Alzheimer 
de Doença de Alzheimer

\begin{tabular}{|c|c|c|}
\hline $\begin{array}{l}\text { Carvalho, } \\
\text { Magalhães \& } \\
\text { Pedroso (2016) }\end{array}$ & $\begin{array}{l}\text { Tratamentos não farmacológicos } \\
\text { que melhoram a qualidade de vida } \\
\text { de idosos com Doença de } \\
\text { Alzheimer: uma revisão sistemática }\end{array}$ & $\begin{array}{l}\text { Realizar uma revisão sistemática sobre quais são os tratamentos não } \\
\text { farmacológicos que ajudam a melhorar a qualidade de vida (QV) de idosos } \\
\text { com doença de Alzheimer (DA) mais descritos na literatura nos últimos dez } \\
\text { anos (2006-2016). }\end{array}$ \\
\hline $\begin{array}{l}\text { Ferreira \& } \\
\text { Massano (2013) }\end{array}$ & $\begin{array}{l}\text { Terapêutica farmacológica na } \\
\text { doença de Alzheimer: progressos e } \\
\text { esperanças futuras }\end{array}$ & $\begin{array}{l}\text { Fazer uma revisão dos progressos alcançados até ao momento presente } \\
\text { relativamente às intervenções terapêuticas farmacológicas na DA, em } \\
\text { particular as que possam ter efeito modificador da doença, bem como os } \\
\text { fármacos em desenvolvimento, pelo que foi efetuada uma pesquisa da } \\
\text { literatura e ensaios clínicos registados em www.clinicaltrials.gov. }\end{array}$ \\
\hline $\begin{array}{l}\text { Kucmansk et al. } \\
\text { (2016) }\end{array}$ & $\begin{array}{l}\text { Doença de Alzheimer: desafios } \\
\text { enfrentados pelo cuidador no } \\
\text { cotidiano familiar }\end{array}$ & $\begin{array}{l}\text { Analisar os desafios enfrentados pelo cuidador no cotidiano familiar de } \\
\text { pacientes com doença de Alzheimer do município de Chapecó, SC. }\end{array}$ \\
\hline $\begin{array}{l}\text { Borghi et al. } \\
(2013)\end{array}$ & $\begin{array}{l}\text { Sobrecarga de familiares } \\
\text { cuidadores de idosos com doença } \\
\text { de Alzheimer: um estudo } \\
\text { comparativo }\end{array}$ & $\begin{array}{l}\text { Comparar a sobrecarga do cuidador familiar principal com a do secundário } \\
\text { do idoso com doença de Alzheimer e identificar qual dimensão gera maior } \\
\text { impacto. }\end{array}$ \\
\hline
\end{tabular}

Brigola et al. Perfil de saúde de cuidadores Analisar o perfil das queixas de saúde de cuidadores e identificar
(2017)
familiares de idosos e sua relação características do cuidado relacionadas em uma população de cuidadores de
com variáveis do cuidado: um um município do interior paulista, área rural do Brasil.
estudo no contexto rural

Queiroz et al. Perfil sociodemográfico e Analisar associação entre o perfil sociodemográfico, características do (2018) qualidade de vida de cuidadores de cuidado e a qualidade de vida de cuidadores de idosos diagnosticados com idosos com demência demência.

\begin{tabular}{|c|c|c|}
\hline $\begin{array}{ll}\text { Seima, } & \text { Lenardt } \\
\& & \text { Caldas } \\
(2014) & \end{array}$ & $\begin{array}{l}\text { Relação no cuidado entre o } \\
\text { cuidador familiar e idoso com } \\
\text { Alzheimer }\end{array}$ & $\begin{array}{l}\text { Interpretar a relação no cuidado entre cuidadores familiares e idosos com } \\
\text { Alzheimer, alicerçada na dialética concreta da participação segundo os } \\
\text { quatro preceitos de Gabriel Marcel. }\end{array}$ \\
\hline $\begin{array}{l}\text { Mendes \& } \\
\text { Santos (2016) }\end{array}$ & $\begin{array}{l}\text { O cuidado na doença de Alzheimer: } \\
\text { as representações sociais dos } \\
\text { cuidadores familiares }\end{array}$ & $\begin{array}{l}\text { Observar e identificar as representações dos cuidadores familiares sobre o } \\
\text { cuidado e analisar como influenciam em suas práticas de cuidado }\end{array}$ \\
\hline
\end{tabular}

Fonte: Dados da pesquisa (2019).

\section{Discussão}

Conforme Pereira, Teixeira e Santos (2012), qualidade de vida é uma variável multifatorial, que abrange desde a estabilidade financeira até critérios de satisfação pessoal, e estabelece estreita relação com a capacidade de autonomia e de independência do indivíduo. No contexto da Doença de Alzheimer (DA), uma demência de caráter progressivo, a incapacidade 
de autocuidado e a perda das habilidades necessárias às atividades cotidianas pelo idoso evidenciam o comprometimento da qualidade de vida dos pacientes e a necessidade de cuidadores para auxílio desse grupo.

Segundo o Ministério da Saúde (2013), foram realizados três estudos no Brasil quanto à análise da predominância da DA em grupos de idosos de base comunitária. Os resultados mostraram que, na população de mais de 65 anos, a DA constituiu a causa de $55 \%$ dos casos de demência.

Conforme Cavalcanti e Engelhardt (2012), a Doença de Alzheimer é uma condição com diferentes causas, associada a condicionantes genéticos, ambientais, metabólicos, distúrbios vasculares e oxidativos, além de reações inflamatórias e alterações mitocondriais. A fisiopatologia da DA associa-se à perda de sinapses e ao acúmulo de peptídeos beta-amiloide no espaço extracelular e à degeneração neurofibrilar devido à agregação da proteína tau, que está vinculada à estabilização de microtúbulos (Cavalcanti \& Engelhardt, 2012).

Por 27 anos o diagnóstico de Alzheimer foi baseado em critérios estabelecidos ainda em 1984, pelo Instituto Nacional do Envelhecimento e Associação de Alzheimer, os quais baseavam-se na sintomatologia e na avaliação do relato do paciente e de seus familiares. Segundo Nitzsche, Moraes \& Júnior (2015), com os avanços no estudo da DA, surgiu a necessidade de atualização desses parâmetros, de modo que os critérios passaram a incluir as três fases do desenvolvimento da DA: préclínico, comprometimento leve e demência; além de exames de neuroimagem, como ressonância magnética e tomografia computadorizada, e biomarcadores.

Trata-se de um diagnóstico essencialmente clínico, com ênfase em alterações cognitivas, comportamentais e funcionais, associado à análise de biomarcadores, investigação laboratorial e neuroimagem (Coelho et al., 2015).

Pacientes com Alzheimer apresentam diferentes manifestações clínicas, de modo que a progressão de cada quadro é variável (Cardoso et al., 2015). Essas manifestações e sua progressão devem sempre ser levadas em consideração, pois através delas os resultados encontrados sobre aspectos de vida, tanto destes pacientes quanto de seus cuidadores, podem ser justificados.

Inicialmente, o acometimento por DA está associado a um quadro de perda da memória e dificuldade de execução de novas tarefas. Com a progressão da doença, ocorre prejuízo de outras funções cognitivas como cálculo e raciocínio além de afasia e apraxia. Já nos estágios terminais da doença, de acordo com Carretta \& Scherer (2012), observam-se modificações no ciclo do sono, alterações comportamentais e comprometimento da marcha bem como da capacidade de autocuidado.

Segundo Ferreira e Massano (2013), no que se refere ao tratamento farmacológico da DA, observa-se que ensaios clínicos vem sendo desenvolvidos para definir a eficácia de novos fármacos diante desse quadro demencial. Embora existam discordâncias, nota-se constante empenho no desenvolvimento de novas alternativas envolvendo agentes como davunetide, azul de metileno, huperzine A, neurotrofinas, insulina intra-nasal, entre outros. Ainda de acordo com Ferreira \& Massano (2013), na literatura há alusões à possibilidade de a eficácia da terapêutica no tratamento da DA estar relacionada ao início precoce das intervenções. Considerando que os idosos com DA geralmente estão polimedicados, por apresentarem outros tratamentos em curso, este estudo também destaca a necessidade de melhor caracterização da farmacocinética dos fármacos diante do risco de interação medicamentosa e de efeitos adversos, já que muitos desses medicamentos não são seletivos nem específicos para o alvo em questão.

Ademais, observa-se que existem também algumas intervenções não farmacológicas disponíveis, a exemplo da arteterapia e da reabilitação cognitiva e multidisciplinar. Destaca-se a importância dessas intervenções multidisciplinares no quadro de Alzheimer, já que são benéficas em nível cognitivo, físico, de relacionamentos sociais e de atividades de vida diária, AVD’s (Carvalho, Magalhães \& Pedroso, 2016).

Mediante o exposto, nota-se que o diagnóstico de Alzheimer tem reflexo em toda a conjuntura familiar. Como se trata de uma doença com muitas implicações, os membros familiares precisam se adequar às necessidades do idoso de modo que tal 
adaptação interfere no bem-estar de todo o núcleo familiar. Considerando a complexidade dessa demência, evidencia-se a necessidade de uma visão ampla sobre a condição do idoso, o qual, em função da perda de memória, de atenção e de percepção no tempo e no espaço (Santos \& Borges, 2015); precisa ser considerado em todas as suas dimensões, e não só do ponto de vista biológico.

Nesse sentido, observa-se que o cuidador tem papel fundamental na assistência domiciliar e pode ser definido como o responsável por auxiliar os idosos com Doença de Alzheimer (DA) em suas atividades diárias, provendo sua subsistência (Neumann \& Dias, 2013). Os cuidadores auxiliam na promoção do bem-estar geral dos idosos com DA, já que participam das intervenções farmacológicas e não farmacológicas. A função de cuidador do paciente acometido por Alzheimer é, frequentemente, atribuída a um familiar, o qual não é previamente habilitado para executar essa responsabilidade de cuidado. No Brasil, esse papel é assumido, de forma preponderante, por esposas e filhas (Folle, Shimizu \& Naves, 2016).

Segundo Kucmanski et al. (2016), existem diferentes classificações para esses cuidadores. Os cuidadores considerados primários são aqueles que fornecem conforto e segurança ao cuidado, auxiliam nas atividades de vida diárias, assim como nas tarefas domésticas. O que difere o cuidador primário do secundário, é que o secundário tem autonomia para tomada de decisões e, com isso, maior nível de responsabilidade. Já os cuidadores terciários são considerados assistentes: auxiliam nas compras e no transporte, recebem pensão e quitam as contas (Kucmanski et al., 2016).

Desse modo, o processo de cuidar em doenças demenciais como o Alzheimer, mostra-se permeado por situações desgastantes já que o modo de vida do cuidador é bastante alterado em função das demandas da pessoa cuidada. Assim, considerando que a DA requer assistência permanente ao idoso, os cuidadores apresentam-se, muitas vezes, sobrecarregados, tanto do ponto de vista físico quanto emocional. Essa rotina desgastante aumenta a possibilidade de os cuidadores desenvolverem problemas de saúde física, como desordens respiratórias e propensão a infecções, bem como acometimentos psíquicos, o que prejudica o autocuidado do familiar que presta assistência (Borghi et al., 2013).

Dentre as patologias que surgem ao longo da vida dos cuidadores de idosos dependentes, pode-se destacar: depressão, ansiedade e estresse (Brigola et al., 2017). Os cuidadores estão mais propensos a esses acometimentos, muitas vezes, em função da falta de suporte e de conhecimento desse grupo (Queiroz et al., 2018). Ressalta-se ainda que a idade avançada dos cuidadores familiares aliada ao desenvolvimento de outras atividades paralelas à responsabilidade de auxiliar o paciente incapacitado, contribuem com esse contexto de sobrecarga (Seima, Lenardt \& Caldas, 2014).

Ademais, a maneira com que os cuidadores compreendem e lidam com os desafios envolvidos no cuidado em casos de DA tem reflexo significativo no processo terapêutico. O processo de cuidar pode ser interpretado com base em diferentes dimensões sociais e suas representações tem reflexo no modo de cuidar (Folle, Shimizu \& Naves, 2016). Segundo Mendes \& Santos (2016), as principais representações quanto ao processo de cuidar, na ótica dos cuidadores, são: cuidado como missão, como desarmonia das identidades sociais, como prisão e como gratidão.

Para os cuidadores que interpretam o cuidar como missão, trata-se de uma responsabilidade entendida como um encargo que não pode ser negado ao parente fragilizado, está vinculado ao laço familiar e ao dever moral. Nessa ótica, o papel de provedor do cuidado é interpretado também como herança cultural, já que é uma função mais desempenhada por mulheres, as quais, do ponto de vista histórico e cultural, são mencionadas como cuidadoras da família (Mendes \& Santos, 2016). Já entre os que consideram essa atribuição resultado da desarmonia das identidades sociais, observa-se a sensação de angústia, ansiedade e desequilíbrio diante da "inversão dos papéis sociais", provocadas pelo quadro demencial. Nesse sentido, o cuidador interpreta que o adoecimento, progressivo e irreversível, ocasiona uma despersonalização dos idosos ao comprometer sua percepção como patriarca/matriarca do núcleo familiar (Mendes \& Santos, 2016).

Conforme Folle, Shimizu e Naves (2016); para alguns cuidadores, a assistência ao idoso com Alzheimer está associada à sensação de prisão. Mediante a necessidade de atendimento integral ao idoso, o qual está incapacitado de realizar 
as atividades diárias, muitos cuidadores abdicam de muitos aspectos de sua rotina para adequar-se às demandas do paciente. Essa abnegação repercute negativamente na vida particular, profissional e social do cuidador.

Outra forma de entender o processo de cuidado é interpretá-lo como gratificante. Para esse grupo de cuidadores, auxiliar o idoso em suas atividades básicas é uma oportunidade de retribuir o cuidado recebido em outras etapas da vida. Nesse sentido, a função não está atrelada a uma obrigação, mas ao vínculo afetivo existente e à sensação de compromisso (Folle, Shimizu \& Naves, 2016).

Trata-se de um diagnóstico que acarreta alterações no convívio familiar, especialmente no que tange ao cuidador primário. Observa-se que perspectivas de tratamento antes negligenciadas vem sendo analisadas sob diferentes enfoques, de modo a incluir o núcleo familiar e os cuidadores como elemento fundamental do processo terapêutico (Carvalho, Magalhães \& Pedroso, 2016). Com frequência, os cuidadores de pacientes com DA executam simultaneamente vários papéis, fato que, aliado ao caráter neurodegenerativo e progressivo da doença, que requer acompanhamento integral dos idosos; contribui com o cotidiano desgastante e exaustivo vivenciados pelos cuidadores. Esse quadro prejudica a qualidade de vida dos familiares bem como dos idosos com DA, o que sinaliza que a família é, além de cuidadora, unidade a ser cuidada (Cesário et al., 2017).

\section{Conclusão}

A análise realizada neste estudo evidenciou que o diagnóstico de Doença de Alzheimer envolve um cenário desafiador, considerando a repercussão exercida na qualidade de vida dos idosos e de seus cuidadores, bem como no modo de cuidar. Observou-se que se trata de uma demência de prevalência significativa, cuja etiologia ainda não está plenamente estabelecida na literatura, e cujo tratamento é alvo de estudos em andamento. Foi visto também que o declínio cognitivo progressivo da doença, aliado à dependência cada vez mais acentuada dos idosos quanto aos cuidadores, acarreta sobrecarga física e mental.

Ademais, os autores relatam que as diversas maneiras pelas quais os cuidadores interpretam e lidam com a assistência prestada aos idosos com DA, interferem no processo de cuidado. É pertinente ressaltar também a necessidade de difusão de informações quanto ao Alzheimer, a exemplo de ações de educação em saúde, para desmistificar alguns aspectos da patologia já que, muitas vezes, o quadro inicial dessa demência é entendido erroneamente como parte do processo de senescência, o que dificulta também o diagnóstico precoce da DA.

Além disso, considerando que a Doença de Alzheimer representa a forma mais comum de demência em idosos, é importante que estudos futuros abordem intervenções educacionais e de suporte social e emocional voltadas ao cuidador, buscando potencializar os conhecimentos e habilidades de quem presta assistência e amenizar o impacto na qualidade de vida dos cuidadores primários que assistem aos idosos com DA. Ressalta-se a necessidade de trabalhos que contemplem medidas que possam ser inseridas e adaptadas ao cotidiano dos cuidadores e familiares diretamente envolvidos no processo de cuidado demandando pela DA. As repercussões desses trabalhos poderão contribuir com as estratégias de promoção de saúde e com o planejamento da terapêutica direcionada à Doença de Alzheimer no sistema de saúde.

Desse modo, a tendência atual de envelhecimento das populações sinaliza a necessidade de um olhar mais holístico e empático para os quadros da geriatria, especialmente, os demenciais; de modo que destaca-se o papel das equipes multiprofissionais no rastreamento precoce e direcionamento de acometimentos como o Alzheimer para as demais instâncias das Redes de Atenção à Saúde.

\section{Referências}

Borghi, A. C., Castro, V. C., Marcon, S. S., \& Carreira L. (2013). Sobrecarga de familiares cuidadores de idosos com doença de Alzheimer: um estudo comparativo. Revista Latino-Americana de Enfermagem, 21 (4). https://www.scielo.br/pdf/rlae/v21n4/pt_0104-1169-rlae-21-04-0876.pdf. 
Brasil. (2013). Ministério da Saúde. Protocolos Clínicos e Diretrizes Terapêuticas. Doença de Alzheimer. Portaria SAS/MS nº 1.298 , de 21 de novembro de 2013.

Brigola, A. G., Luchesi, B. M., Rossetti, E. S., Mioshi, E., Inouye, K., \& Pavarini, S. C. I. (2017). Perfil de saúde de cuidadores familiares de idosos e sua relação com variáveis do cuidado: um estudo no contexto rural. Revista Brasileira de Geriatria e Gerontologia, 20 (3). ttp://dx.doi.org/10.1590/198122562017020.160202.

Cardoso, V. B., Silva, J. L. A., Dutra, C. D. C., Tebaldi, J. B., \& Costa, F. A. M. M. (2015). A doença de alzheimer em idosos e as consequências para cuidadores domiciliares. Revista Memorialidades, 12 (23, 24), 113-149. https://periodicos.uesc.br/index.php/memorialidades/article/view/1310.

Carretta, M. B., \& Scherer, S. (2012). Perspectivas atuais na prevenção da Doença de Alzheimer. Estudos Interdisciplinares sobre o Envelhecimento, 17 (1), 37-57. https://seer.ufrgs.br/RevEnvelhecer/article/view/14368/23187.

Carvalho, P. D. P., Magalhães, C. M. C., \& Pedroso, J. S. (2016). Tratamentos não farmacológicos que melhoram a qualidade de vida de idosos com doença de Alzheimer: uma revisão sistemática. Jornal Brasileiro de Psiquiatria, 65 (4), 334-339. https://doi.org/10.1590/0047-2085000000142.

Cavalcanti, J. L. S., \& Engelhardt E. (2012) Aspectos da fisiopatologia da doença de Alzheimer esporádica. Revista Brasileira de Neurologia, 48 (4), $21-29$. http://files.bvs.br/upload/S/0101-8469/2012/v48n4/a3349.pdf.

Cesário, V. A. C., Leal, M. C. C., Marques, A. P. O., \& Claudino, K. A. (2017). Estresse e qualidade de vida do cuidador familiar de idoso portador da doença de Alzheimer. Revista Saúde em Debate, 41 (112), 171-182. http://dx.doi.org/10.1590/0103-1104201711214.

Coelho G. G., Teixeira P. S., Novak J. C., \& Galhardi M. (2015). Os impactos na saúde do cuidador familiar de pessoas com doença de Alzheimer: uma revisão bibliográfica. Revista Ciência e Estudos Acadêmicos de Medicina, 3, 57-71. https://periodicos.unemat.br/index.php/revistamedicina/article/view/411.

Ferreira, S., \& Massano. J. (2013). Terapêutica farmacológica na doença de Alzheimer: progressos e esperanças futuras. Arquivos de Medicina, 27 (2), 65-86. http://www.scielo.mec.pt/scielo.php?script=sci_arttext\&pid=S0871-34132013000200004\&ln g=pt.

Folle, A. D., Shimizu, H. E., \& Naves, J. O. S. (2016). Representação social da doença de Alzheimer para familiares cuidadores: desgastante e gratificante. Revista da Escola de Enfermagem da USP, 50 (1), 81-87. https://doi.org/10.1590/S0080-623420160000100011.

Kucmanski, L. S., Zenevicz, L., Geremia, D. S., Madureira, V. S. F., Silva, T. G., \& Souza, S. S. (2016). Doença de Alzheimer: desafios enfrentados pelo cuidador no cotidiano familiar. Revista Brasileira de Geriatria e Gerontologia, 19 (6), 1022-1029. https://doi.org/10.1590/1981-22562016019.150162.

Mendes, C. F. M., \& Santos, A. L. S. (2016). O cuidado na doença de Alzheimer: as representações sociais dos cuidadores familiares. Revista Saúde e Sociedade, 25 (1), 121-132. https://doi.org/10.1590/S0104-12902015142591.

Menezes, M. R., Alves, M. B., Souza, A. S., Silva, V. A., Silva, E. N., \& Oliveira, C. M. S. (2013). Comportamento agressivo na relação entre idoso e cuidador familiar em doenças demenciais. Ciência Cuidado e Saúde, 12 (4), 744-751. https://doi.org/10.4025/cienccuidsaude.v12i4.19529.

Neumann, S. M. F., \& Dias, C. M. S. B. (2013). Doença de Alzheimer: o que muda na vida do familiar cuidador?. Revista Psicologia e Saúde, 5 (1), $10-17$. http://pepsic.bvsalud.org/scielo.php?script=sci_arttext\&pid=S2177-093X2013000100003\&lng=pt.

Nitzsche, B. O., Moraes, H. P., \& Júnior, A. R. T. (2015) Doença de Alzheimer: novas diretrizes para o diagnóstico. Revista Médica de Minas Gerais, 25 (2), 237-243. http://www.dx.doi.org/10.5935/2238-3182.20150043.

Pereira, A. S., Shitsuka, D. M., Parreira, F. J., \& Shitsuka, R. (2018). Metodologia da pesquisa científica. Universidade Federal de Santa Maria. https://repositorio.ufsm.br/bitstream/handle/1/15824/Lic_Computacao_Metodologia-Pesquisa-Cientifica.pdf?sequence=1.

Pereira, E. F., Teixeira, C. S., \& Santos, A. (2012). Qualidade de vida: abordagens, conceito e avaliação. Revista Brasileira de Educação Física e Esporte, 26 (2), 241-250. https://doi.org/10.1590/S1807-55092012000200007.

Qiu C. (2012). Prevenção da Doença de Alzheimer visando fatores de risco vascular: esperança e lacuna. Jornal Doença de Alzheimer, 32 (3), 721 - 731. https://pubmed.ncbi.nlm.nih.gov/22842870/.

Queiroz, R. S., Camacho, A. C. L. F., Gurgel, J. L., Assis, C. R. C., Santos, L. M., \& Santos, M. L. S. C. (2018). Perfil sociodemográfico e qualidade de vida de cuidadores de idosos com demência. Revista Brasileira de Geriatria e Gerontologia, 21 (2), 210-219. http://dx.doi.org/10.1590/1981-22562018021.170170.

Santos, M. D., Borges, \& S. M. (2015). Percepção de funcionalidade nas fases leve e moderada da doença de Alzheimer: visão do paciente e seu cuidador. Revista Brasileira de Geriatria e Gerontologia, 18 (2), 339-349. https://doi.org/10.1590/1809-9823.2015.14154.

Seima, M. D., Lenardt, M. H., \& Caldas, C. P. (2014). Relação no cuidado entre o cuidador familiar e o idoso com Alzheimer. Revista Brasileira de Enfermagem, 67 (2), 233-240. https://doi.org/10.5935/0034-7167.20140031. 